\title{
Disentangling input and output-related components of spatial neglect
}

\author{
Tobias Loetscher ${ }^{1,2 *}$, Michael E. R. Nicholls ${ }^{1}$, Amy Brodtmann ${ }^{3}$, Nicole A. Thomas ${ }^{1}$ and \\ Peter Brugger ${ }^{2,4}$
}

1 School of Psychology, Flinders University, Adelaide, SA, Australia

2 Department of Neurology, University Hospital Zurich, Zurich, Switzerland

${ }^{3}$ Florey Neuroscience Institutes, Melbourne, Australia

${ }^{4}$ Zurich Center for Integrative Human Physiology (ZIHP), Zurich, Switzerland

\section{Edited by:}

Mario Bonato, University of Padova, Italy

Reviewed by:

Mario Bonato, University of Padova, Italy

A. M. Barrett, Kessler Foundation, USA

*Correspondence:

Tobias Loetscher, School of Psychology, Flinders University, GPO Box 2100, Adelaide, 5001 SA, Australia.

e-mail: tobias.loetscher@ alumni.ethz.ch
Spatial neglect is a heterogeneous disorder with a multitude of manifestations and subtypes. Common clinical paper and pencil neglect tests fail to differentiate between these subtypes. For example, neglect patients typically bisect lines to the right. This bias can be caused by an underestimation of the left half of the line (input-related deficit), by the failure to direct actions toward the left side of space (output-related deficit), or by a mixture of these impairments. To disentangle these impairments, we used a test consisting of a line bisection task on a touch screen monitor (manual motor task) and the subsequent judgment of one's own bisection performance (visual perceptual task). It was hypothesized that patients with mainly output-related neglect should be better able to recognize their misbisected lines than patients with purely input-related neglect. In a group of 16 patients suffering from spatial neglect after right brain damage, we found that patients were three times more likely to suffer from a predominantly input-related than from an output-related subtype. The results thus suggest that neglect is typically an input-related impairment. Additional analysis of the line bisection task revealed that temporal (slowness in initiation and execution of contralateral movements) and spatial (insufficient movement amplitude toward the contralesional side) aspects of output-related neglect were mutually unrelated. This independence raises the possibility that a fine-grained differentiation of output-related neglect is required. That is, impairments in lateralized temporal and spatial aspects of movements may underlie different neglect subtypes.

\footnotetext{
Keywords: spatial neglect, rehabilitation, neglect subtype, motor neglect, perceptual neglect, attention, proof of concept, stroke
}

\section{INTRODUCTION}

Spatial neglect is a disabling disorder of lateralized cognition and behavior (Bradshaw and Mattingley, 1995). It is characterized by a failure to report, respond, or orient to stimuli presented to the side opposite a brain lesion, which cannot be attributed to elementary sensory-motor impairments (Heilman, 1979). The presence of neglect is associated with an unfavorable prognosis in terms of rehabilitation outcome, length of hospital stay, and daily living activities after discharge to home (Robertson and Halligan, 1999; Nys et al., 2005). Knowledge of the processes guiding spontaneous recovery and effective therapeutic approaches for spatial neglect is very sparse (Bowen and Lincoln, 2007a,b). This is surprising, given that spatial neglect occurs in a substantial number of patients suffering from brain damage (about $45 \%$ of patients after right and $20 \%$ of patients after left brain damage, see Bowen et al., 1999). There is, therefore, an obvious clinical need for a better understanding of the cognitive mechanisms underlying spatial neglect to advance therapeutic interventions.

A fundamental difficulty in advancing neglect therapy derives from the inherent heterogeneity of the disorder. Numerous subtypes and forms of spatial neglect have been described in the literature (Bartolomeo and Chokron, 2001; Buxbaum et al., 2004; Barrett et al., 2006). Some patients with right brain damage, for example, may primarily neglect the left side of their body (personal neglect), whereas other patients neglect the left in reaching (near) space, and still others only neglect the left side of space beyond their reach (far space, see Halligan and Marshall, 1991; Vuilleumier et al., 1998). Furthermore, some patients may be particularly affected when detecting left-sided stimuli, while others are impaired during the initiation, execution and/or aiming of motor responses toward those stimuli (Heilman, 1979, 2004). The wide variety of behavioral neglect profiles is commonly thought to originate from damage to distinct neural substrates in a widely distributed cortical network, which mediates attention in the brain (Mesulam, 1999; Verdon et al., 2010). It is conceivable, therefore, that therapeutic success could be improved if treatments are targeted to the specific pattern of the impairment. In support of this view, there is preliminary evidence that patients with different forms of neglect may respond differently to specific treatments (Barrett et al., 1999, 2001; Adair et al., 2003). 
While the potential importance of isolating neglect subtypes is emphasized in authoritative reviews on neglect therapy (Barrett et al., 2006; Bowen and Lincoln, 2007a), standard clinical tests, such as line bisection, copying or cancellation tasks, all fail to provide information on the subtypes of neglect. For example, while a marked rightward deviation in the bisection of horizontal lines indicates left-sided neglect, the reason for this deviation can differ between patients. Some patients may deviate to the right because of a perceptual-attentional bias toward the right-side (inputrelated deficit) while others deviate to the right because of a failure to direct actions toward the left side of space (output-related aiming deficit). There may also be individuals with a mixture of input and output impairments, which makes them difficult to sort neatly into either category (Bisiach et al., 1990; Schwartz et al., 1999; Bartolomeo and Chokron, 2001). Performance on the traditional line bisection task, however, does not allow the clinician to distinguish between these possibilities.

A number of techniques have been devised to disentangle input- and output-related accounts of neglect. The general rationale in many of these techniques is to uncouple the direction of hand movements (output) from the location of the corresponding visual target (input). One study used, for example, a horizontal pulley device with a pointer mounted on the upper pulley string (Bisiach et al., 1990). Participants were asked to move the pointer to the midpoint of a line. In one condition, participants grasped the pointer on the upper string and moved it to the subjective midpoint. The directions of hand and pointer movements were therefore congruent. In a second condition, participants controlled the pointer by moving the lower pulley string. Consequently, hand and pointer movements were in opposite directions: pulling the lower string leftwards resulted in rightward pointer movements, and vice-versa, when the lower string was moved rightward. Neglect was classified to be predominantly input-related if an identical rightward deviation was found in both conditions. Conversely, output-related neglect was indicated by marked leftward deviations in the incongruent pulley condition, because impairments in pulling the lower pulley string leftwards resulted in a failure to shift the pointer toward the right. Other studies have reported similar perceptual/motor dissociations using devices such as reversing mirrors (Tegner and Levander, 1991), video cameras (Na et al., 1998), and overhead projectors (Nico, 1996) to present left/right mirror-reversed visual feedback on a display. These techniques also allow the assessment of input and output-related biases, not only in clinical populations, but also in healthy (Garza et al., 2008; Fortis et al., 2011) and aged participants (Chen et al., 2011).

While the ingenuity of the above techniques is not disputed, the interpretation of the data is not quite as straight-forward as one might wish. Some researchers have pointed out that coordinating spatially incongruent movements from visual feedback is highly confusing and some patients might simply fail to handle the high task-demands (Mattingley et al., 1998; Husain et al., 2000; Vallar, 2001). That is, deficits in the execution of mirror-reversed movements are not necessarily exclusive to output-related deficits of neglect, but might also derive from frontal executive dysfunctions in resolving the cognitive conflict between movement direction and visual feedback (Fink et al.,
1999). Such conflicts might particularly arise during the initial phases of learning motor responses to incongruent visual feedback. Once the new motor skills are fully acquired frontal executive conflicts might be less common as motor responses are now thought to be implicit and automatic (Halsband and Lange, 2006).

A paradigm that does not involve conflicting visuo-motor movements is the passive line bisection task. In this task, patients observe an experimenter as he/she moves the tip of a pen along a line. The patient then indicates verbally when the subjective midpoint has been reached by the pen's tip (Reuter-Lorenz and Posner, 1990). However, the use of moving stimuli (see also Halligan and Marshall, 1989; Chiba et al., 2006 for related methods) is not ideal because the stimuli may act as a visual cue and cues are well known to modulate spatial neglect (Riddoch and Humphreys, 1983). The paradigm may therefore not provide an uncontaminated indication of input-related neglect.

A compelling alternative to the use of a moving marker is the presentation of pre-bisected lines ("landmark task", Milner et al., 1993; Harvey et al., 1995). The landmark task requires patients to point to the end of the line closer to the transaction mark. Patients with input-related neglect are assumed to perceive the left-side of accurately bisected lines as shorter and to point to that side. Conversely, those patients consistently pointing to the right side are thought to suffer from output-related neglect. Harvey et al. (1995) required neglect patients to carry out a relatively difficult landmark task where the bisector was placed only up to $5 \mathrm{~mm}$ to the left or right of the true middle. While the ability of the task to detect input-related neglect is not disputed, the task's sensitivity to output-related neglect has been questioned (Husain et al., 1998; Harvey, 2004). Bisiach et al. (1998) modified the landmark task used by Harvey et al. (1995) using stimuli where the difference between the left and right halves of the line ranged from 30 to $150 \mathrm{~mm}$. By making the task easier, Bisiach et al. (1998) identified more patients with output-related neglect. That said, because the task was easy, it was less able to identify patients with input-related neglect. Thus, while it appears that the ideal placement of the bisection mark is an unsettled issue, analyses involving curve fitting procedures have been proposed to circumvent this problem (Toraldo et al., 2002, 2004). In any case, when reviewing the strength and weaknesses of the different tasks developed for assessing input and output-related tasks, Harvey (2004, p. 327) concluded that landmark tasks are "the most appropriate, thoroughly researched tool" for such assessments.

While patients with pure output-related neglect are impaired in reaching to the left-side of space they are thought to have relatively spared perceptual skills. Asking patients to judge their own bisections therefore offers a solution to shed light on the underlying impairment. Based on this reasoning, the first goal of the current study was to introduce a simple method of disentangling input- and output-related neglect. In traditional versions of the landmark task, the experimenter bisects the lines and then presents them to the patient. This may not be ideal because of the difficulties in determining which level of difficulty to use and because the lines are not related to the patient's actual motor behavior in space. Both these issues can be solved if the patients generate the stimuli themselves. Our new experimental paradigm 
first requires the patient to bisect a series of horizontal lines. Subsequently, the lines are presented again and the patient judges their own bisections (see Figure 1). By asking the patient to bisect a line and then judge that bisection, the task should allow us to disentangle input- and output-related neglect. Output-related impairments hamper the placement of the bisection mark at the intended location, but not the subsequent perceptual error judgment task. Accordingly, patients with output-related neglect were expected to recognize their own bisection errors. Patients with input-related neglect were expected to show a different pattern of results. Because their output is relatively spared, their manual bisections were expected to be subjectively accurate. Therefore, when judging the accuracy of their own bisections, they were expected to have much more difficulty.

Output-related neglect may not only affect the spatial scale of goal-directed movements but also the timing of these movements. That is, patients with output-related neglect may be impaired in initiating leftward movements (directional hypokinesia) and/or may be slowed in the execution of leftward movements (directional bradykinesia). Impairments in these temporal aspects of goal-directed movements were first observed in animal studies (Watson et al., 1978) and have subsequently been described in neglect patients (Heilman et al., 1985; Mattingley et al., 1992, 1994). Investigations of temporal impairments in movements are commonly investigated using simple reaction time paradigms where the initiation and response times for movements toward left- and right-sided targets are compared (Heilman et al., 1985; Mattingley et al., 1992; Husain et al., 2000; Buxbaum et al., 2004).

Although the neural processes underlying the timing of movement may be distinct from those involved in scaling the movement amplitude (Mattingley et al., 1992, 1994), the relationship between temporal and spatial characteristics of output-related neglect has received relatively little attention in the literature. The second goal of the study is to examine the behavioral relationship between the temporal and spatial aspects of output-related neglect. Specifically, the time required to perform leftward relative to rightward bisections is compared to the spatial deviations of leftward relative to rightward bisections. A correlation between these measures would imply that differentiating between the two

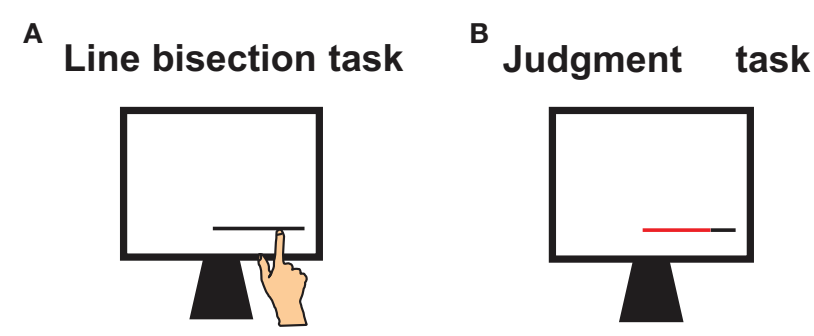

FIGURE 1 | Tasks and procedure. (A) Participants first performed a line bisection task in which they pointed to the lines' midpoint with their index finger. (B) Participants then judged their own bisection errors. Each line, divided into two colored segments at the locations where the participant previously placed the bisection mark, was then re-presented. Participants had to name the color of the longer line segment. subtypes of output-related neglect is not necessary. Conversely, independence of temporal and spatial biases implies that a fine-grained differentiation of what constitutes output-related neglect is needed.

\section{MATERIALS AND METHODS PARTICIPANTS}

Sixteen patients with left-sided spatial neglect after right brain damage ( 12 men, mean age 62 years, SD $=11$ years, see Table 1 for demographic and clinical details) participated in this study. Like our previous study, inclusion criteria were based on the presence of a right hemisphere lesion and signs of left-sided neglect for at least two out of five standard paper and pencil tests. Tests included line bisection, cancellation, figure copying, reading, and a figural fluency task (see Loetscher and Brugger, 2009 for details and cut-off criteria). Visual field deficits were assessed clinically by finger perimetry. Twenty healthy participants ( 11 men, mean age 61 years, SD $=14$ years $)$ matched for age and years of education served as controls. The study protocol was performed in accordance with the ethical standards laid down in the Declaration of Helsinki and all subjects gave written informed consent.

\section{APPARATUS AND PROCEDURE}

Stimuli were presented on an Elo Entuitive 17' LCD desktop touch monitor $\left(15,500\right.$ touchpoints $\left./ \mathrm{cm}^{2}\right)$. The generation and sequencing of stimuli was controlled with Java Script programming. Participants were seated with their midsagittal plane aligned to the center of the touch screen. The viewing distance was roughly $560 \mathrm{~mm}$; eye level was slightly above the vertical center of the screen. Eye and limb movements were not constrained.

The participants were first familiarized with the touch screen. They performed a pointing task, in which they pointed as accurately as possible to different illuminated circles on the screen. All participants were able to do so. After familiarization, the participants performed the line bisection task and subsequently the error judgment task (see Figure 1).

\section{Line bisection task}

In the line bisection task, participants pointed with their right index finger to the subjective middle of horizontal lines presented on the screen. The presentation of a line was triggered by pressing the space bar with the right index finger. After pointing to the subjective midpoint, the screen turned blank (white) and the space bar had to be pressed again to start the next trial. With this procedure a central starting point for each trial was assured.

In total, 18 black lines with a length of $160 \mathrm{~mm}$ were presented in a pseudo-randomized order either on the left, center or right side of the screen. There were six lines per side of presentation with the lines in the lateral conditions being shifted $40 \mathrm{~mm}$ to the left and right, respectively. The time period between pressing the space bar (elicitation of the line) and the touch response on the screen, as well as the horizontal coordinates of each fingertip were recorded. There were no time constraints for the response. The deviation of the these coordinates from the objective midpoint of the line were measured to the nearest millimeter-with right-sided errors scored as positive and left-sided errors as negative deviations. 
Table 1 | Demographic, clinical, and neuropsychological details of neglect patients with right brain damage.

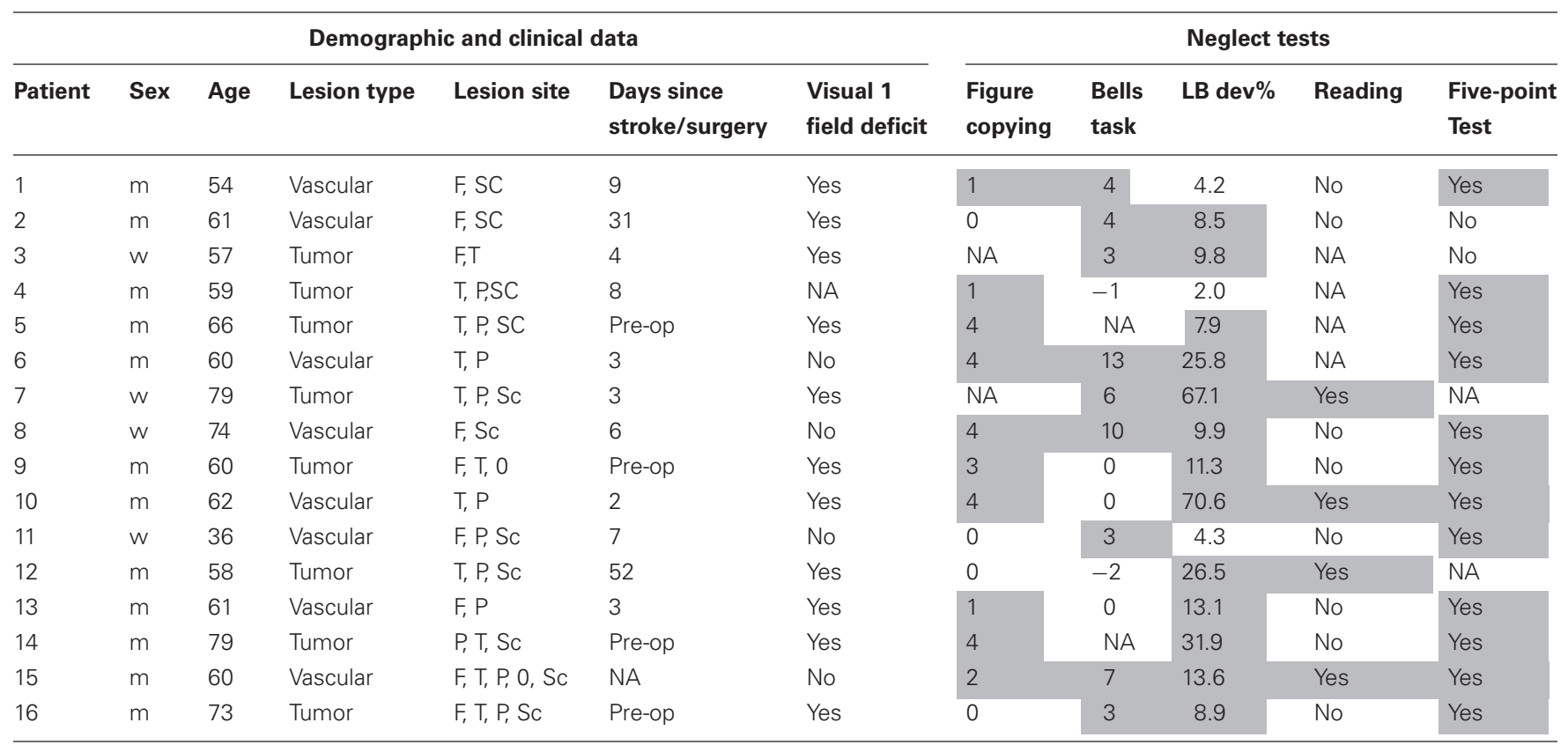

Lesion site: $F=$ Frontal, $T=$ Temporal, $P=$ Parietal, $O=$ Occipital, $S C=$ Subcortical; Days since surgery/operation: number indicates days since stroke or operation, pre-op = preoperative.

Neglect tests: (A) Figure copying, scores range from 0 (no omissions) to 4 [several left-sided omissions, see Azouvi et al. (2002) for details], cut-off point >0; (B) "Bells task" (Gauthier et al., 1989), score gives number of left minus right-sided omissions, cut-off point $>2$; (C) LB dev\% $=$ Line Bisection deviation in \% from true half, positive values denote rightward deviations, cut-off point > 6.5; (D) Reading, cut-off point > 0 left-sided omission; (E) five-point-test = figural fluency task (Regard et al., 1982), cut-off point > O omission of left-sided columns. Cut-off scores for the neglect tests A-D as defined in Azouvi et al. (2002); for test $E$ as in Vuilleumier et al. (2004). Tests above cut-off-point are gray-colored. NA = Not available.

\section{Error judgment task}

In the line judgment task, each of the previously bisected 18 lines were presented in sequence at the same location as the line bisection task. The lines were divided into two segments at the point where the participant previously placed the bisection mark. The left and right segments were colored red or black, respectively. In order to counterbalance the coloring of the lines, each line was presented twice, once with the left side colored black (right side red) and once with the left side red (right side black). The 36 lines were presented in a pseudo-randomized order and participants named the color of the longer line segment (forced choice design with no time constraints for response). The subject's task was to say which line segment was longer ("red" or "black") and verbal responses were recorded by the examiner. The percentage of correct judgments was the dependent variable.

\section{RESULTS}

\section{LINE BISECTION TASK}

Data were collapsed across the side presentation (left, center, right). As the data in the neglect group were not normally distributed (Shapiro-Wilk $p<0.01$ ) non-parametric tests were applied. Wilcoxon signed-rank tests were used to determine whether the bisection biases were significantly different from zero. Patients with neglect bisected the lines too far to the right side (median $4.9 \mathrm{~mm} ; Z=2.8, p<0.006$ ), while healthy controls showed a leftward bias (median $=-2.5 \mathrm{~mm}$;
$Z=3.2, p<0.002)$. An independent-samples Mann-Whitney test demonstrated a significant difference between the neglect and control groups $(U=37, Z=3.9, p<0.001)$.

Subgroup analyses found no difference between patients with and without visual field deficits $(U=18.0, Z=0.5, p=$ n.s.) and patients with vascular and tumor etiology $(U=32.0$, $Z<0.01, p=$ n.s.).

\section{ERROR JUDGMENT TASK}

Neglect patients (median correct judgments $72.2 \%$ ) were significantly worse in detecting their own bisection errors compared to the controls (median 93.5\%; $U=51.5, Z=3.5, p<0.001$ ). The standard deviation was more than four times larger in the neglect group (SD 25.2) compared to controls (SD 5.7), indicating considerable heterogeneity in the neglect population.

The number of correct judgments was not modulated by the presence of visual field deficits $(U=20.5, Z=0.2, p=$ n.s. $)$, or lesion etiology $(U=31.0, Z=0.1, p=$ n.s. $)$.

\section{DISENTANGLING INPUT AND OUTPUT-RELATED COMPONENTS}

To extricate input- and output-related components, cut-off criteria for the line bisection and error judgment task were defined. For the line bisection task, Bayesian inferential statistics were used to determine the cut-off scores, which differentiated the controls from neglect patients (see Crawford and Garthwaite, 2007). Using this technique, deviations larger than $2.4 \mathrm{~mm}$ 
were classified as significantly different from controls (Bayesian $p<0.05)$.

For the error judgment task, cut-off scores were based on normative data collected in an unpublished pilot study with 71 healthy subjects. That study showed that the accuracy in error judgments depended on the magnitude of the bisection error. That is, larger errors were much easier to spot than smaller ones (see Figure 2). The varying degree of difficulty in judging errors was controlled by calculating the lower bound of a $95 \%$ confidence interval for the percentage of correct error judgments for each bisection deviation. The corresponding values were then fitted with a cumulative normal distribution function. This curve fitting procedure determined a cut-off score for the percentage of correct judgment as a function of bisection error. Judgment scores below the lower bound confidence curve were considered to reflect an input-related impairment.

Using the cut-off criteria outlined above, individual patient data were plotted in Figure 3 to distinguish input and outputrelated components of spatial neglect. Inspection of Figure 3 allows a number of conclusions to be drawn: (1) The bisection and judgment performance of four neglect patients was within normal limits; (2) three patients showed signs of neglect in the bisection task, but intact judgment abilities. These patients can be considered as suffering from output-related neglect; (3) nine patients were impaired in the bisection and judgment task. These patients can be considered as suffering predominantly, but not necessarily exclusively, from input-related neglect; (4) the performance of neglect patients is characterized by considerable heterogeneity compared to the rather homogenous performance of controls.

\section{RELATIONSHIP BETWEEN TEMPORAL AND SPATIAL BIASES IN LINE BISECTION}

To investigate the relationship between temporal (time differences in bisecting left and right-sided lines) and spatial aspects (differences in deviation errors for left and right-sided lines) lateralization scores were calculated (Bryden and Sprott, 1981).
The temporal lateralization score was calculated as the natural logarithm of the ratio "bisection times of lines presented on the left side divided by bisection times of lines presented on the right side." Positive values denote quicker responses to right-sided lines, negative values indicate an advantage for left-sided lines, and a value of zero denotes equal response times for left and right-sided lines. A spatial bias measure was calculated using an analogous procedure. As the logarithm has to be drawn from positive values, the use of deviation measures was not appropriate. Instead, the positions of the bisection mark as measured from the left end of the line were used. The spatial lateralization score was then calculated as the natural logarithm of the ratio "bisection position of lines presented on the right side divided by the bisection position of lines presented on the left side." Here, negative values denote that left-sided lines are bisected further to the right than right-sided lines (vice-versa for positive values).

The relationship between temporal and spatial aspects of neglect is plotted in Figure 4. The two aspects were uncorrelated in neglect patients $(r=-0.15, p=$ n.s. $)$ and controls $(r=0.12$, $p=$ n.s.).

\section{DISCUSSION}

Common tests of neglect, such as line bisection and cancellation tasks, fail to provide information on the subtype of neglect that is present. The primary goal of the current study was to introduce a simple method that allows us to dissociate input- and output-related subtypes of neglect. This method required patients to bisect a series of horizontal lines (manual motor task) and then to judge their own bisections (visual perceptual task). In both of these tasks, typical signs of neglect emerged at a group level. That is, a rightward deviation in the line bisection task (Schenkenberg et al., 1980) and impairments in detecting horizontal length asymmetries in the perceptual judgment task (Milner et al., 1993). The results in the neglect group were also characterized by large standard deviations, signifying considerable heterogeneity in performance. Such an increased variability in performance has been

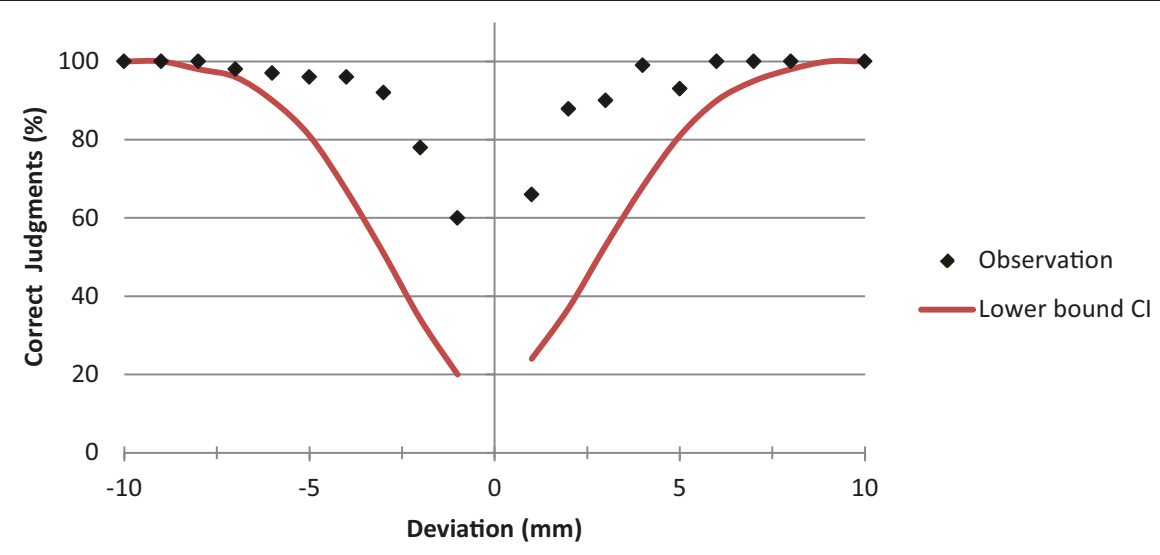

FIGURE 2 | Dependency of correct judgment on deviation error.

The percentage of correct judgments is plotted as a function of bisection error. The graph shows the normative data of 71 healthy subjects (black dots) and the corresponding lower bound of the 95\% confidence interval (red line) Judgment scores below the lower bound of the fitted confidence interval curve were considered as indicating impaired perceptual judgment abilities. 


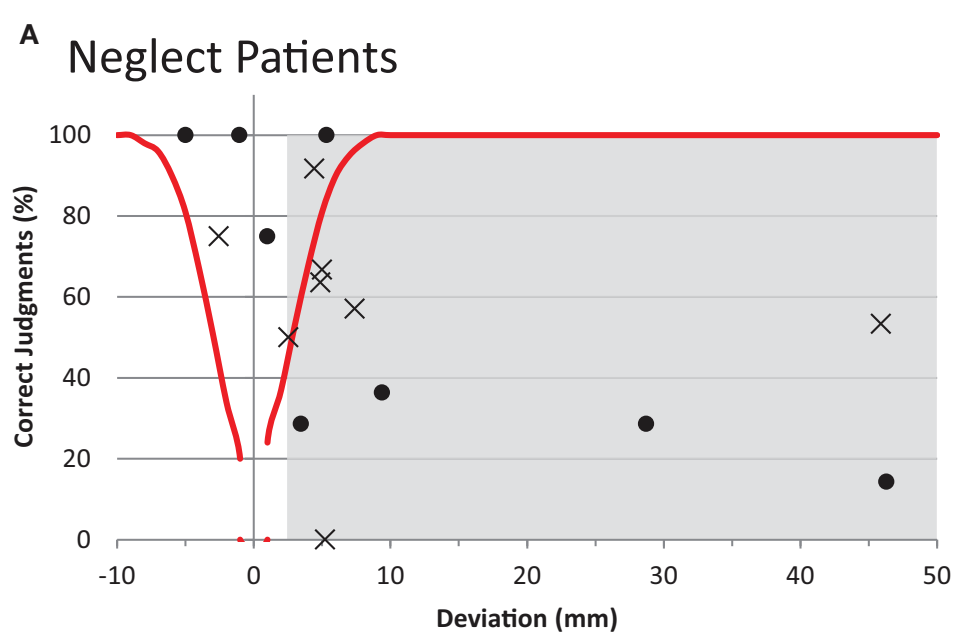

${ }^{B}$ Controls

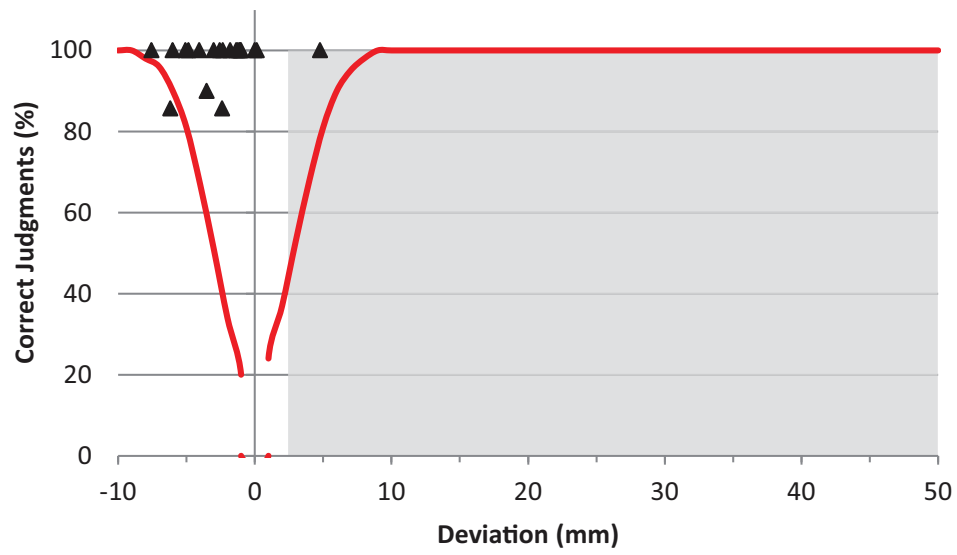

LB Neglect

Judgment cut-off

- Tumor Patients

$\times$ Vascular Patients

$\Delta$ Controls
FIGURE 3 | Disentangling input- and output-related neglect.

(A) Shows the performance of the individual patients in the line bisection and error judgment task (split by etiology). Bisection values in the gray colored area indicate the presence of neglect in the line bisection task.
Error judgments below the red curve indicate impaired perceptual judgment abilities. See main text for indications of input or output-related neglect (B) Shows the corresponding performance of the control participants. shown to be a valid marker of neglect (Marshall and Halligan, 1989; Bonato et al., 2008).

The extrication of input- and output-related subtypes was based on individual performances in the two tasks. With the emphasis being the identification of the predominant subtype, the 16 neglect patients could be classified in three distinct subgroups (see also Figure 3). The first group, comprising three patients, showed a rightward bias when bisecting lines, but no difficulties in recognizing the erroneous bisections when judging them. This pattern clearly corresponds to an output-related deficit, which is indicated by impairments in movements toward the left (resulting in rightward deviations) and intact spatial perceptual capabilities. The second group of nine patients demonstrated neglect in the bisection and the perception task. Intact spatial perception allows the patient to detect misplaced bisection marks. An inability to do so would therefore indicate a perceptual impairment. These patients were accordingly classified as suffering predominantly, but not necessarily exclusively, from an input-related deficit. The third group, comprising the remaining four patients, showed normal performances in the line bisection and judgment task and therefore could not be classified as having input- or outputrelated neglect. It should be noted, however, that an inability to isolate an input/output subtype in some patients is not necessarily due to a lack of test sensitivity. Indeed, input/output subtypes of neglect are just one dimension along which neglect patients can differ (Barrett et al., 2006). Furthermore, the inclusion criterion for neglect in the current study was based on signs of neglect in at least two out of five common paper and pencil tests. A rightward deviation for the line bisection task was therefore not a precondition for inclusion.

The current study revealed that patients with neglect were three times more likely to suffer from a predominantly input-related than from an output-related subtype. While this proportion gives some indication of the relative incidence of input- and output-related neglect, it should be borne in mind that the patient sample used in the current study was relatively small. The relative incidence of the different subtypes of neglect is further complicated by the different methods of assessment. 


\section{A}

\section{Neglect Patients}

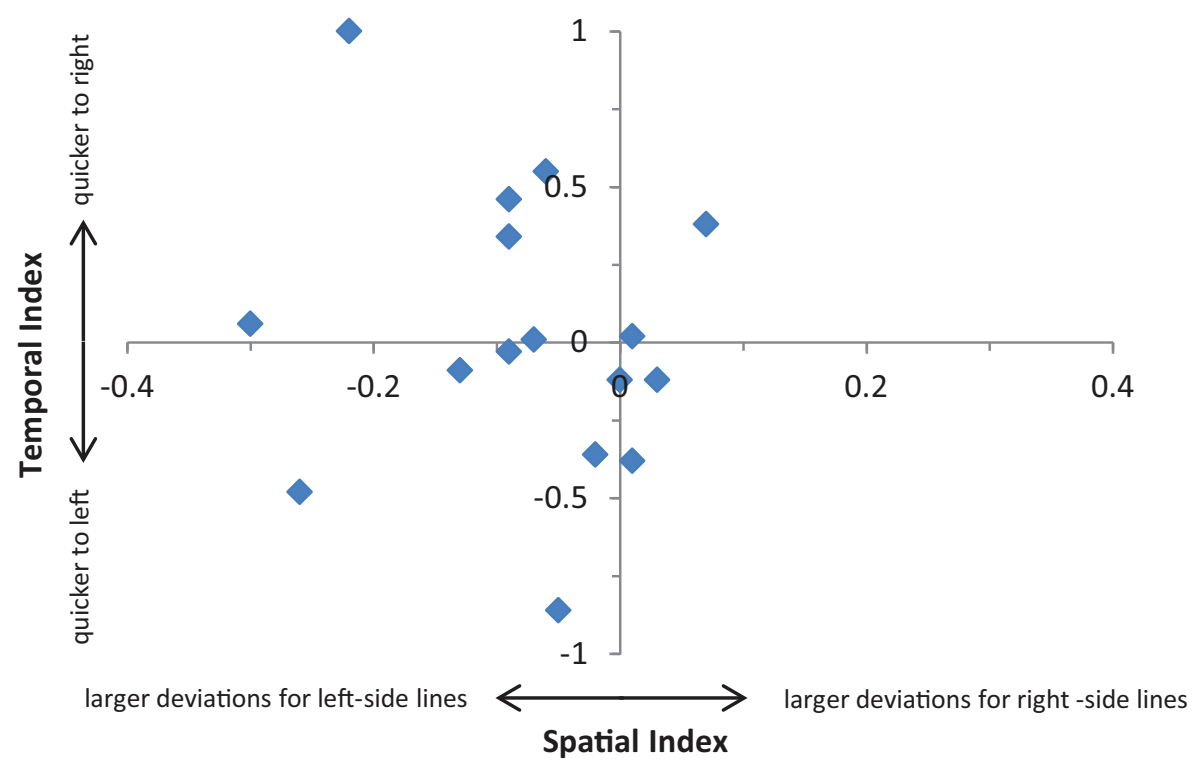

B

\section{Controls}

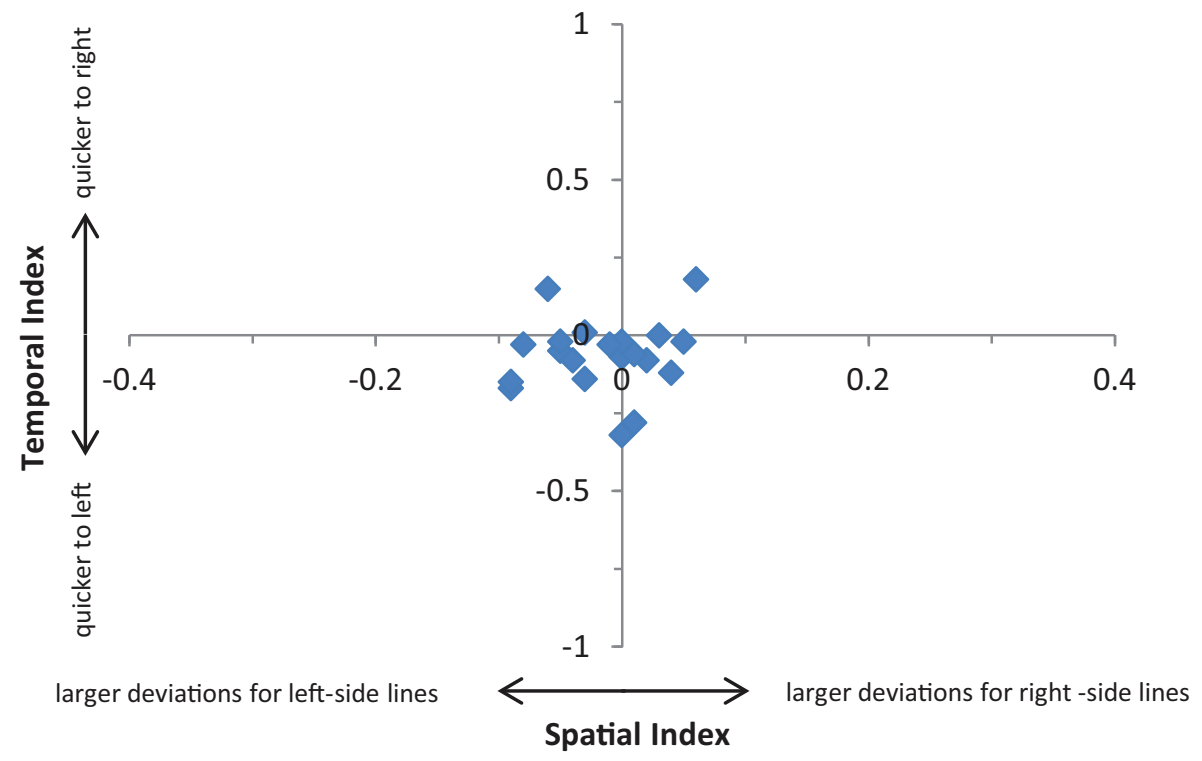

FIGURE 4 | Relationship between temporal and spatial aspects of neglect. (A) Shows 23 individual patients' temporal (i.e., time required to perform leftward relative to rightward 24 bisections) and spatial (i.e., deviations of leftward relative to rightward bisections) scores 25 in the line bisection task. See main text for details (B) Shows the corresponding 26 performance of the control participants.
For example, inconsistent classifications have been observed between the landmark and the pulley tests (Harvey et al., 2002) and line bisection and cancellation tests (Adair et al., 1998; $\mathrm{Na}$ et al., 1998). Bearing these points in mind, the relative proportion of the input/output subtypes observed in the current study should be treated with some caution. Nevertheless, the finding of more patients with input-related neglect is consistent with almost all previous studies assessing these subtypes in larger patient samples ( $n>40$, Mijovic, 1991; Buxbaum et al., 2004; Shimodozono et al., 2006; Sapir et al., 2007). The current results only stand in contrast to one large patient sample study $(n=121$, Bisiach et al., 1998), which reported a higher proportion of output-related 
neglect for a landmark bisection task. In this case, however, the landmark stimuli were easy to discriminate, with a difference of at least $30 \mathrm{~mm}$ between the left and right segments of the line. Because of the easy perceptual nature of this task, it is likely that patients with mild or moderate input-related neglect were able to detect the difference-resulting in a low relative incidence of that form of neglect.

A secondary goal of the study was to compare spatial and temporal aspects of output-related neglect. Our results indicate that these aspects are independent. Impairments in the initiation and execution of leftward movements were not associated with a marked rightward deviation in the line bisection task. Despite the problems of drawing implications from null results, the findings suggest that a fine-grained differentiation of outputrelated neglect is required. That is, it is conceivable that temporal and spatial aspects constitute different neglect subtypes. The phenomenological differences could be based on distinct neural processes underlying the timing of movement and the scaling of the movement amplitude (Mattingley et al., 1992, 1994). If this holds true, then attempts to elucidate the anatomical substrates of output-related neglect should differentiate temporal and spatial measures. Neglecting this sort of differentiation might have contributed to the controversy regarding the role of the parietal lobe in motor neglect (as addressed, for example, in Carey, 1998; Husain et al., 1998). While many studies assessing motor impairments by spatial measures found a predominance of anterior impairments (e.g., Bisiach et al., 1990; Coslett et al., 1990; Nico, 1996; Na et al., 1998), it is noteworthy that the studies which found that posterior brain regions were associated with motor neglect applied temporal measurers (Mattingley et al., 1998; Husain et al., 2000;but see Sapir et al., 2007 for contradictory finding). It remains to be seen to what degree this discrepancy is related to the use of different measures. In any case, the independence of spatial and temporal measures observed in the current study clearly demonstrates that these aspects would deserve a more fine-grained analysis in the literature.

The results of the current have demonstrated the feasibility of our new method of dissociating input- and output-related neglect, which has some important advantages over previous methods. One advantage is that it does not involve conflicting visuo-motor movements. This is important as there are anecdotal reports of patients who refused to continue with incongruent movement tests because they found them too frustrating (Nico, 1996). Such task requirements have accordingly been criticized as being too confusing for patients with brain damage (Mattingley et al., 1998; Husain et al., 2000). In contrast, the current paradigm is straightforward and relatively easy-even for patients with severe forms of neglect. The current method also addresses some potential shortcomings of the landmark test, which is still considered to be one of the best tools for differentiating input- and output-related forms of neglect (Harvey, 2004). A major issue for the landmark test is the placement of the bisector and the subsequent difficulty of the task. Bisiach et al. (1998) proposed, for example, the use of stimuli in which the difference between the left and right segments of the line ranged from 30 to $150 \mathrm{~mm}$. As discussed above, it is doubtful that these easy discriminations would identify subtle forms of perceptual neglect. The optimal transector placement might, in fact, depend on the severity of the patient's impairment. A second problem with the landmark task is that they are required to judge spatial relations, which are not derived from their motor behavior. Contrasting a patient's actual motor behavior with the subsequent judgment of this behavior seems to be a more intuitive and natural way to infer subtypes of neglect.

While the methods introduced in the study address some of the weaknesses of previous research, the study has some limitations on its own. First, the task does not necessarily provide a pure measure of output-related impairments. The manual line bisection task involves both motor and visual input components. It is therefore possible that the paradigm biased the findings toward input over output-related deficits (see Garza et al., 2008; Chen et al., 2011). It should also be noted that the bisection task and the visual perceptual task may differ with respect to the involvement of bottom-up (exogenous) and top-down (endogenous) processes. That is, judging the accuracy of bisected lines might have involved more top-down, endogenous orienting processes than the line bisection task. As deficits in endogenous and exogenous orienting can be dissociated in patients (Bartolomeo and Chokron, 2002; Sieroff et al., 2007; Loetscher et al., 2010), it is possible that differences in the processing demands of the tasks affected the results.

The assessment of neurological impairments and activities in everyday functions with scales such as the Barthel Index (Mahoney and Barthel, 1965) and the Catherine Bergego Scale (Azouvi et al., 2003) may have provided a better characterization of patients. A better functional characterization might have been important as there is some evidence, for example, that items of the Catherine Bergego Scale are better predictors of output- versus input-related neglect impairments than performance in standard neglect tests (Goedert et al., 2012). It is noteworthy that the patient characteristics that were assessed, such as lesion etiology and visual field deficits, yielded inconclusive results. While these characteristics did not affect task performances statistically, the sample of 16 neglect patients might simply have been too small to uncover any differences. Hemianopia, for example, has been shown to influence line bisection tasks (e.g., Doricchi and Angelelli, 1999; Doricchi et al., 2002) and this influence may vary depending on the time since stroke (Saj et al., 2012). Clearly, a sample of just four patients without visual field deficits, as in the current study, cannot address the modulating effect of hemianopia conclusively. Importantly, however, an exclusion of the four patients (three with inputrelated and one with output-related deficits) does not change the main results: there was still is a predominance of inputover output-related subtypes. The same predominance is also evident when considering patients with vascular etiology only (see Figure 3). The findings in the tumor group are somewhat different as three out of eight tumor patients performed within normal limits. However, when only considering patients affected by one of the two investigated subtypes, there was also a clear predominance of input (four patients) over outputrelated subtypes (one patient). To summarize, the current study has observed a predominance of input-related neglect across 
a variety of patient characteristics. While this predominance appears to be consistent, it should be borne in mind that broader conclusions are limited by the relatively small patient sample.

Although the heterogeneity of the sample probably added some noise to the data, including patients with different etiologies can also be seen as an advantage. Different methodological problems and confounds are associated with specific etiologies (Bartolomeo, 2011). Thus, studies combining a variety of etiologies, such as stroke and tumor data, might provide converging evidence on the widely distributed functional brain network that controls spatial attention.

While neglect patients typically exhibit an ipsilesional bias, some patients may show signs of a paradoxical contralesional bias (Robertson et al., 1994). What is more, ipsilesional and contralesional biases may be dissociated with respect to input- and output-related errors (Bisiach et al., 1990; Schwartz et al., 1999; Barrett and Burkholder, 2006). While potentially only a minority of patients would show such a dissociation, it should be acknowledged that the present method is not sensitive to uncover these patients.

Finally, it should be recognized that the assumption of a strict dichotomy between input and output-related aspects of neglect is likely to be a considerable oversimplification (Adair et al., 1998). Planning, executing, and visually guiding movements toward targets rely on continuous integration of sensory information. As a result, input and output-related processing streams will interact-resulting in a blurring of the boundaries between the processes (Mesulam, 1981, 1999). From a strictly theoretical perspective, therefore, it might be more accurate to describe the relationship between the two neglect subtypes along a continuum instead of a strict dichotomy. It is also likely that both types of deficit are present to varying degrees in an individual patient. The current method of analysis allows the determination of the predominant subtype only. While this determination might be useful in a clinical context (see below), it is worth considering refining the method and analysis in future studies to obtain continuous and mathematically independent measures of input versus output-related deficits. The methodological approach for analysing landmark task performance proposed by Toraldo and colleagues $(2002,2004)$ could serve as a template for improving the current task. The approach involves psychometric curve fitting procedures and illustrates nicely how mathematical modeling of behavior can help improving clinical measures.

\section{REFERENCES}

Adair, J. C., Na, D. L., Schwartz, R. L., and Heilman, K. M. (1998). Analysis of primary and secondary influences on spatial neglect. Brain Cogn. 37, 351-367.

Adair, J. C., Na, D. L., Schwartz, R. L., and Heilman, K. M. (2003). Caloric stimulation in neglect: evaluation of response as a function of neglect type. J. Int. Neuropsychol. Soc. 9, 983-988.

Azouvi, P., Olivier, S., de Montety, G., Samuel, C., Louis-Dreyfus, A., and

From a clinical and rehabilitative perspective, however, the input/output dichotomy might be useful. There are suggestions, for example, that patients who present with a predominantly output-related subtype are more likely to have a chronic disability (Eskes and Barrett, 2009; Goedert et al., 2012) and input-related impairments seem especially amenable to recovery (Rengachary et al., 2011). Different treatment regimens may also affect input and output-related neglect differently. This idea has been examined for several interventions by one laboratory (e.g., with monocular patching: Barrett et al., 2001, 2004; Barrett and Burkholder, 2006; Chen et al., 2009). Intuitively, it seems plausible that perceptual-attentional impairments and deficiencies in spatial movements respond best to different treatment approaches. Rehabilitation procedures like attentional alerting, scanning training, or monocular patching may be more efficacious for input than output-related neglect. Conversely, procedures like limb activation therapy and dopaminergic medications may be more appropriate for the treatment of output-related neglect (see Barrett et al., 2006; Sapir et al., 2007 for comprehensive discussion of treatment rationales).

While the diagnosis of the subtypes of neglect is potentially important for subsequent treatment (Barrett et al., 2006; Bowen and Lincoln, 2007a), there are still a number of unanswered questions. Foremost amongst these is the issue of the stability of neglect subtypes over time. To our knowledge, only one study has addressed this issue (Hamilton et al., 2007). In that study, subtypes were assessed in 21 acute neglect patients at three different time points separated by at least one week. Eighteen of those patients $(86 \%)$ showed significant variability in their performance on measures of neglect subtype. The authors claim that this inconsistency was not related to spontaneous recovery or practice effects. It is not clear, at present, whether the results are due to methodological limitations (e.g., insufficient sensitivity or specificity of the tests) or fluctuations in individual neglect behavior. If the latter proposition turns out to be correct, then this would have implications for the validity of tests of neglect subtypes and for the prognosis and treatment of those subtypes. The testing paradigm used in the current study may help to shed light on this issue.

\section{ACKNOWLEDGMENTS}

We thank Enrique Wintsch, Nicole Kavan, and Pascale Sandmann for programming assistance and help in collecting normative data.

of spatial neglect after right hemisphere stroke. J. Neurol. Neurosurg. Psychiatry 73, 160-166.

Barrett, A. M., and Burkholder, S. (2006). Monocular patching in subjects with right-hemisphere stroke affects perceptual-attentional bias. J. Rehabil. Res. Dev. 43, 337-345.

Barrett, A. M., Buxbaum, L. J., Coslett, H. B., Edwards, E., Heilman, K. M., Hillis, A. E., Milberg, W. P., and Robertson, I. H. (2006). Cognitive rehabilitation interventions for neglect and related disorders: moving from bench to bedside in stroke patients. J. Cogn. Neurosci. 18, 1223-1236.

Barrett, A. M., Crucian, G. P., Beversdorf, D. Q., and Heilman, K. M. (2001). Monocular patching may worsen sensory-attentional neglect: a case report. Arch. Phys. Med. Rehabil. 82, 516-518.

Barrett, A. M., Crucian, G. P., and Heilman, K. M. (2004). Eye patching biases spatial attention after thalamic hemorrhage in a patient without spatial neglect: a case 
report. Arch. Phys. Med. Rehabil. 85, 1017-1020.

Barrett, A. M., Crucian, G. P., Schwartz, R. L., and Heilman, K. M. (1999). Adverse effect of dopamine agonist therapy in a patient with motorintentional neglect. Arch. Phys. Med. Rehabil. 80, 600-603.

Bartolomeo, P. (2011). The quest for the 'critical lesion site' in cognitive deficits: problems and perspectives. Cortex 47, 1010-1012.

Bartolomeo, P., and Chokron, S. (2001). "Levels of impairment in unilateral neglect," in Disorders of Visual Behavior, 2nd Edn. ed M. Behrmann (Amsterdam: Elsevier Science), 67-98.

Bartolomeo, P., and Chokron, S. (2002). Orienting of attention in left unilateral neglect. Neurosci. Biobehav. Rev. 26, 217-234.

Bisiach, E., Geminiani, G., Berti, A., and Rusconi, M. L. (1990). Perceptual and premotor factors of unilateral neglect. Neurology 40, 1278-1281.

Bisiach, E., Ricci, R., Lualdi, M., and Colombo, M. R. (1998). Perceptual and response bias in unilateral neglect: two modified versions of the Milner landmark task. Brain Cogn. 37, 369-386.

Bonato, M., Priftis, K., Marenzi, R., and Zorzi, M. (2008). Modulation of hemispatial neglect by directional and numerical cues in the line bisection task. Neuropsychologia 46, 426-433.

Bowen, A., and Lincoln, N. B. (2007a). Cognitive rehabilitation for spatial neglect following stroke. Cochrane Database Syst. Rev. doi: 10.1002/ 14651858.CD003586.pub2

Bowen, A., and Lincoln, N. B. (2007b). Rehabilitation for spatial neglect improves test performance but not disability. Stroke 38, 2869-2870.

Bowen, A., McKenna, K., and Tallis, R. C. (1999). Reasons for variability in the reported rate of occurrence of unilateral spatial neglect after stroke. Stroke 30, 1196-1202.

Bradshaw, J. L., and Mattingley, J. B. (1995). Clinical Neuropsychology. San Diego, CA: Academic Press.

Bryden, M. P., and Sprott, D. A. (1981). Statistical determination of degree of laterality. Neuropsychologia 19, 571-581.

Buxbaum, L. J., Ferraro, M. K., Veramonti, T., Farne, A., Whyte, J., Ladavas, E., Frassinetti, F., and Coslett, H. B. (2004). Hemispatial neglect: subtypes, neuroanatomy, and disability. Neurology 62, 749-756.
Carey, D. P. (1998). Action, perception, cognition, and the inferior parietal cortex. Trends Cogn. Sci. 2, 162-164.

Chen, P., Erdahl, L., and Barrett, A. M. (2009). Monocular patching may induce ipsilateral "where" spatial bias. Neuropsychologia 47, 711-716.

Chen, P., Goedert, K. M., Murray, E., Kelly, K., Ahmeti, S., and Barrett, A. M. (2011). Spatial bias and right hemisphere function: sexspecific changes with aging. J. Int Neuropsychol. Soc. 17, 455-462.

Chiba, Y., Yamaguchi, A., and Eto, F. (2006). Assessment of sensory neglect: a study using moving images. Neuropsychol. Rehabil. 16, 641-652.

Coslett, H. B., Bowers, D., Fitzpatrick, E., Haws, B., and Heilman, K. M. (1990). Directional hypokinesia and hemispatial inattention in neglect. Brain 113, 475-486.

Crawford, J. R., and Garthwaite, P. H. (2007). Comparison of a single case to a control or normative sample in neuropsychology: development of a Bayesian approach. Cogn Neuropsychol. 24, 343-372.

Doricchi, F., and Angelelli, P. (1999). Misrepresentation of horizontal space in left unilateral neglect: role of hemianopia. Neurology 52, 1845-1852.

Doricchi, F., Galati, G., Deluca, L., Nico, D., and D'Olimpio, F. (2002). Horizontal space misrepresentation in unilateral brain damage. I. Visual and proprioceptive-motor influences in left unilateral neglect. Neuropsychologia 40, 1107-1117.

Eskes, G. A., and Barrett, A. M. (2009). "Neuropsychological Rehabilitation," in Neurovascular Neuropsychology, eds R. Lazar and J. Festa (New York, NY: Springer), 281-305.

Fink, G. R., Marshall, J. C., Halligan, P. W., Frith, C. D., Driver, J., Frackowiak, R. S. J., and Dolan, R. J. (1999). The neural consequences of conflict between intention and the senses. Brain 122, 497-512.

Fortis, P., Goedert, K. M., and Barrett, A. M. (2011). Prism adaptation differently affects motor-intentional and perceptual-attentional biases in healthy individuals. $\mathrm{Neu}$ ropsychologia 49, 2718-2727.

Garza, J. P., Eslinger, P. J., and Barrett, A. M. (2008). Perceptualattentional and motor-intentional bias in near and far space. Brain Cogn. 68, 9-14.

Gauthier, L., Dehaut, F., and Joanette, Y. (1989). The bells test - a quantitative and qualitative test for visual neglect. Int. J. Clin. Neuropsychol. 11, 49-54.
Goedert, K. M., Chen, P., Botticello, A., Masmela, J. R., Adler, U., and Barrett, A. M. (2012). Psychometric evaluation of neglect assessment reveals motor-exploratory predictor of functional disability in acutestage spatial neglect. Arch. Phys. Med. Rehabil. 93, 137-142.

Halligan, P. W., and Marshall, J. C. (1989). Two techniques for the assessment of line bisection in visuo-spatial neglect: a single case study. J. Neurol. Neurosurg. Psychiatry 52, 1300-1302.

Halligan, P. W., and Marshall, J. C. (1991). Left neglect for near but not far space in man. Nature 350, 498-500.

Halsband, U., and Lange, R. K. (2006). Motor learning in man: a review of functional and clinical studies. J. Physiol. Paris 99, 414-424.

Hamilton, R. H., Coslett, H. B., Buxbaum, L. J., Whyte, J., and Ferraro, M. K. (2007). Inconsistency of performance on neglect subtype tests following acute right hemisphere stroke. J. Int. Neuropsychol. Soc. 14, 23-32.

Harvey, M. (2004). Perceptual and premotor neglect: is there an ideal task to categorise patients? Cortex 40 , 323-328.

Harvey, M., Kramer-McCaffery, T., Dow, L., Murphy, P. J. S., and Gilchrist, I. D. (2002). Categorisation of 'perceptual' and 'premotor' neglect patients across different tasks: is there strong evidence for a dichotomy? Neuropsychologia 40, 1387-1395.

Harvey, M., Milner, A. D., and Roberts, R. C. (1995). An investigation of hemispatial neglect using the Landmark Task. Brain Cogn. 27, 59-78.

Heilman, K. M. (1979). "Neglect and related disorders," in Clinical Neuropsychology, eds K. M. Heilman and E. Valenstein (New York, NY: Oxford University Press), 268-307.

Heilman, K. M. (2004). Intentional neglect. Front. Biosci. 9, 694-705.

Heilman, K. M., Bowers, D., Coslett, H. B., Whelan, H., and Watson, R. T. (1985). Directional hypokinesia: prolonged reaction times for leftward movements in patients with right hemisphere lesions and neglect. Neurology 35, 855-859.

Husain, M., Mattingley, J., Rorden, C., Kennard, C., and Driver, J. (1998). Response from husain, mattingley, rorden, kennard and driver. Trends Cogn. Sci. 2, 164-166.

Husain, M., Mattingley, J. B., Rorden, C., Kennard, C., and Driver, J. (2000). Distinguishing sensory and motor biases in parietal and frontal neglect. Brain 123, 1643-1659.

Loetscher, T., and Brugger, P. (2009). Random number generation in neglect patients reveals enhanced response stereotypy, but no neglect in number space. Neuropsychologia 47, 276-279.

Loetscher, T., Nicholls, M. E. R., Towse, J. N., Bradshaw, J. L., and Brugger, P. (2010). Lucky numbers: spatial neglect affects physical, but not representational, choices in a Lotto task. Cortex 46, 685-690.

Mahoney, F. I., and Barthel, D. W. (1965). Functional evaluation: the Barthel Index. Md. State Med. J. 14, 61-65.

Marshall, J. C., and Halligan, P. W. (1989). When right goes left - an investigation of line bisection in a case of visual neglect. Cortex 25, 503-515.

Mattingley, J. B., Bradshaw, J. L., and Phillips, J. G. (1992). Impairments of movement initiation and execution in unilateral neglect. Directional hypokinesia and bradykinesia. Brain 115, 1849-1874.

Mattingley, J. B., Husain, M., Rorden, C., Kennard, C., and Driver, J. (1998). Motor role of human inferior parietal lobe revealed in unilateral neglect patients. Nature 392, 179-182.

Mattingley, J. B., Phillips, J. G., and Bradshaw, J. L. (1994). Impairments of movement execution in unilateral neglect: a kinematic analysis of directional bradyskinesia. Neuropsychologia 32, 1111-1134.

Mesulam, M. (1981). A cortical network for directed attention and unilateral neglect. Ann. Neurol. 10, 309-325.

Mesulam, M. (1999). Spatial attention and neglect: parietal, frontal and cingulate contributions to the mental representation and attentional targeting of salient extrapersonal events. Philos. Trans. R. Soc. Lond. B Biol. Sci. 354, 1325-1346.

Mijovic, D. (1991). Mechanisms of visual spatial neglect. Brain 114, 1575-1593.

Milner, A. D., Harvey, M., Roberts, R. C., and Forster, S. V. (1993). Line bisection errors in visual neglect: misguided action or size distortion? Neuropsychologia 31, 39-49.

Na, D. L., Adair, J. C., Williamson, D. J. G., Schwartz, R. L., Haws, B., and Heilman, K. M. (1998). Dissociation of sensory-attentional from motor-intentional neglect. J. Neurol. Neurosurg. Psychiatry 64, 331-338.

Nico, D. (1996). Detecting directional hypokinesia: the epidiascope 
technique. Neuropsychologia 34, 471-474.

Nys, G. M. S., van Zandvoort, M. J. E., de Kort, P. L. M., van Der Worp, H. B., Jansen, B. P. W., Algra, A., de Haan, E. H. F., and Kappelle, L. J. (2005). The prognostic value of domain-specific cognitive abilities in acute first-ever stroke. Neurology 64, 821-827.

Regard, M., Strauss, E., and Knapp, P. (1982). Children's production on verbal and non-verbal fluency tasks. Percept. Mot. Skills 55, 839-844.

Rengachary, J., He, B. J., Shulman, G., and Corbetta, M. (2011). A behavioral analysis of spatial neglect and its recovery after stroke. Front. Hum. Neurosci. 5:29. doi: 10.3389/fnhum.2011.00029

Reuter-Lorenz, P. A., and Posner, M. I. (1990). Components of neglect from right-hemisphere damage: an analysis of line bisection. Neuropsychologia 28, 327-333.

Riddoch, M. J., and Humphreys, G. W. (1983). The effect of cueing on unilateral neglect. Neuropsychologia 21, 589-599.

Robertson, I., and Halligan, P. (1999). Spatial Neglect: a Clinical Handbook for Diagnosis and Treatment. Hove: Psychology Press.

Robertson, L. H., Halligan, P. W., Bergego, C., Homberg, V.,
Pizzamiglio, L., Weber, E., and Wilson, B. A. (1994). Right neglect following right hemisphere damage? Cortex 30, 199-213.

Saj, A., Honoré, J., Braem, B., Bernati, T., and Rousseaux, M. (2012). Time since stroke influences the impact of hemianopia and spatial neglect on visual-spatial tasks. Neuropsychology 26, 37-44.

Sapir, A., Kaplan, J. B., He, B. J., and Corbetta, M. (2007). Anatomical correlates of directional hypokinesia in patients with hemispatial neglect. J. Neurosci. 27, 4045-4051.

Schenkenberg, T., Bradford, D. C., and Ajax, E. T. (1980). Line bisection and unilateral visual neglect in patients with neurologic impairment. Neurology 30, 509-517.

Schwartz, R. L., Barrett, A. M., Kim, M., and Heilman, K. M. (1999). Ipsilesional intentional neglect and the effect of cueing. Neurology 53, 2017-2022.

Shimodozono, M., Matsumoto, S., Miyata, R., Etoh, S., Tsujio, S., and Kawahira, K. (2006). Perceptual, premotor and motor factors in the performance of a delayed-reaching task by subjects with unilateral spatial neglect. Neuropsychologia 44, 1752-1764.

Sieroff, E., Decaix, C., Chokron, S., and Bartolomeo, P. (2007).
Impaired orienting of attention in left unilateral neglect: a componential analysis. Neuropsychology 21 94-113.

Tegner, R., and Levander, M. (1991). Through a looking glass. a new technique to demonstrate directional hypokinesia in unilateral neglect. Brain 114, 1943-1951.

Toraldo, A., McIntosh, R. D., Dijkerman, H. C., and Milner, A. D. (2002). Disentangling perceptual and response bias in unilateral neglect: a methodological proposal. Brain Cogn. 48, 593-597.

Toraldo, A., McIntosh, R. D. Dijkerman, H. C., and Milner, A. D. (2004). A revised method for analysing neglect using the landmark task. Cortex 40, 415-431.

Vallar, G. (2001). Extrapersonal visual unilateral spatial neglect and its neuroanatomy. Neuroimage 14 S52-S58.

Verdon, V., Schwartz, S., Lovblad, K.-O., Hauert, C.-A., and Vuilleumier, P. (2010). Neuroanatomy of hemispatial neglect and its functional components: a study using voxelbased lesion-symptom mapping. Brain 133, 880-894.

Vuilleumier, P., Ortigue, S., and Brugger, P. (2004). The number space and neglect. Cortex 40, 399-410.
Vuilleumier, P., Valenza, N., Mayer, E., Reverdin, A., and Landis, T. (1998). Near and far visual space in unilateral neglect. Ann. Neurol. 43, 406-410.

Watson, R. T., Miller, B. D., and Heilman, K. M. (1978). Nonsensory neglect. Ann. Neurol. 3, 505-508.

Conflict of Interest Statement: The authors declare that the research was conducted in the absence of any commercial or financial relationships that could be construed as a potential conflict of interest.

Received: 15 February 2012; accepted: 29 May 2012; published online: 13 June 2012.

Citation: Loetscher T, Nicholls MER, Brodtmann A, Thomas NA and Brugger $P$ (2012) Disentangling input and output-related components of spatial neglect. Front. Hum. Neurosci. 6:176. doi: 10.3389/fnhum.2012.00176

Copyright (c) 2012 Loetscher, Nicholls, Brodtmann, Thomas and Brugger. This is an open-access article distributed under the terms of the Creative Commons Attribution Non Commercial License, which permits non-commercial use, distribution, and reproduction in other forums, provided the original authors and source are credited. 